\title{
Prolonged preservation of nerve function in diabetic neuropathy in mice by herpes simplex virus-mediated gene transfer
}

\author{
M. Chattopadhyay • M. Mata • J. Goss $\cdot$ D. Wolfe • \\ S. Huang • J. C. Glorioso • D. J. Fink
}

Received: 5 February 2007 / Accepted: 3 April 2007 / Published online: 17 May 2007

(C) Springer-Verlag 2007

\begin{abstract}
Aims/hypothesis The aim of this study was to determine whether prolonged expression of neurotrophin-3 (NT-3) in mice, achieved by herpes simplex virus (HSV)-mediated gene transfer with gene expression under the control of an HSV latency promoter, can provide protection against the progression of diabetic neuropathy over a 6 month period. Materials and methods Mice with diabetes induced by streptozotocin were inoculated s.c. into both hind feet with a non-replicating HSV vector containing the coding sequence for NT-3 under the control of the HSV latencyassociated promoter 2 (LAP2) elements or with a control vector. Nerve function was evaluated by electrophysiological and behavioural measures over the course of 6 months after the onset of diabetes.

Results Animals inoculated with the NT-3-expressing vector, but not animals inoculated with control vector, showed preservation of sensory and motor nerve amplitude and conduction velocity measured electrophysiologically, small fibre sensory function assessed by withdrawal from heat, autonomic function measured by pilocarpine-induced sweating, skin innervation assessed by protein gene product
\end{abstract}

M. Chattopadhyay $\cdot$ M. Mata $\cdot$ D. J. Fink $(\bowtie)$

Department of Neurology, University of Michigan Health System,

1500 East Medical Center Drive, Room 1914 TC,

Ann Arbor, MI 48109 0316, USA

e-mail: djfink@umich.edu

M. Chattopadhyay $\cdot$ M. Mata $\cdot$ D. J. Fink

Neurology Service, VA Ann Arbor Healthcare System,

Ann Arbor MI, USA

J. Goss $\cdot$ D. Wolfe $\cdot$ S. Huang $\cdot$ J. C. Glorioso

Department of Molecular Genetics and Biochemistry,

University of Pittsburgh,

Pittsburgh, PA, USA
9.5 staining of axons, and density of calcitonin gene-related peptide terminals in the spinal cord measured by immunohistochemistry 5.5 months after vector inoculation.

Conclusions/interpretation These results indicate that the continuous production of NT-3 by LAP2-driven expression of the transgene from an HSV vector over a 6 month period protects against progression of diabetic neuropathy in mice, and provide a proof-of-principle demonstration for the development of a novel therapy for preventing the progression of diabetic neuropathy.

Keywords Diabetes · Diabetic neuropathy - Gene therapy HSV $\cdot$ Neurotrophin-3 · Polyneuropathy

$\begin{array}{ll}\text { Abbreviations } \\ \text { CGRP } & \text { calcitonin gene-related peptide } \\ \text { DRG } & \text { dorsal root ganglia } \\ \text { HSV } & \text { herpes simplex virus } \\ \text { LAP2 } & \text { latency-associated promoter } 2 \\ \text { LAT } & \text { latency-associated transcript } \\ \text { NGF } & \text { nerve growth factor } \\ \text { NT-3 } & \text { neurotrophin-3 } \\ \text { PGP 9.5 } & \text { protein gene product 9.5 } \\ \text { STZ } & \text { streptozotocin } \\ \text { VEGF } & \text { vascular endothelial growth factor }\end{array}$

Introduction

Peripheral polyneuropathy is a common complication of diabetes occurring in up to $50 \%$ of patients. Conversely, diabetes is the most common cause of neuropathy in the Western world and while strict glycaemic control slows progression of neuropathy, no effective treatments are 
available. The pathophysiological basis of nerve damage is complex, involving the formation of reactive oxygen species and the accumulation of advanced glycation endproducts, and modification of the extracellular matrix and blood vessels resulting in nerve ischaemia $[1,2]$. There is circumstantial evidence to indicate that there may be a deficit in neurotrophic factor production in the pathogenesis of diabetic neuropathy [3]; neurotrophin-3 (NT-3) production in muscle and expression of mRNA transcripts for the high affinity NT-3 receptor TrkC in dorsal root ganglia (DRG) neurons are both reduced in rats with diabetic neuropathy [4]. Elevation of NT-3 RNA in nerve and DRG has been suggested to represent an insufficient compensatory response [5], and similar results have been observed in the skin of patients with diabetic neuropathy [6]. Independently of whether the NT-3 deficit plays a pathogenic role in diabetic neuropathy, treatment with NT-3 has been shown to ameliorate many of the deficits in diabetic nerve. Sensory and motor nerve conduction velocity deficits, abnormalities in neurofilament accumulation, mitochondrial dysfunction and activation of stress-related kinases can all be normalised in rodent models by treatment with NT-3 [79] in studies up to 3 months of diabetes. Exogenous administration of other peptide neurotrophic or neuroprotective agents, including nerve growth factor (NGF) [10], erythropoietin [11] and vascular endothelial growth factor (VEGF) [12] also protect nerve structure and function in diabetic neuropathy.

Neurotrophin therapy for human diabetic neuropathy has not been possible, in large part because systemic administration of short-lived potent bioactive peptides to humans is limited by the effects of high doses of these peptides on cells outside the peripheral nervous system that result in intolerable or unacceptable side-effects of administration [13, 14]. Gene transfer provides one means of achieving prolonged production of bioactive macromolecules in vivo to obtain the desired therapeutic effect while avoiding unwanted or untoward side-effects. In several different models, i.m. injection of a lipid-encapsulated plasmid, or adenoviral or adeno-associated viral vectors coding for a neurotrophic or neuroprotective peptide, has been shown to prevent the progression of neuropathy resulting from the administration of neurotoxic drugs or from diabetes [15-17] up to 6 weeks.

In previous work we have demonstrated that s.c. inoculation of a non-replicating herpes simplex virus (HSV)-based vector to transduce neurons of the DRG can be used to express neurotrophic factors in those cells to prevent progression of neuropathy caused by administration of pyridoxine or cisplatin [18-20]. In a mouse model of streptozotocin (STZ)-induced type 1 diabetes, we demonstrated that s.c. inoculation of HSV-based vectors expressing NGF [21] or VEGF [22] 2 weeks after inoculation of STZ can prevent development of neuropathy measured
6 weeks later. These proof-of-principle studies confirm the effectiveness of this mode of HSV-mediated gene transfer, but the time course in those studies was short.

The duration of transgene expression from HSV vectors depends upon the promoter element used to drive transgene expression [23]. In previous studies we used a vector with the human cytomegalovirus immediate early promoter (HCMV IEp) driving NGF expression or the HSV ICP0 gene promoter driving VEGF expression. More recently we have demonstrated that the HSV latency-associated promoter 2 (LAP2) provides prolonged biologically active transgene expression in the central nervous system in models of Parkinson's disease [24], and that the amount of transgene product produced more than 5 months after inoculation of the vector is sufficient to protect against the toxic effects of a subacute overdose of pyridoxine administered 5.5 months after the vector [25]. There have been no prior studies to demonstrate the prolonged effectiveness of gene transfer in the face of continued disease. In the current study we hypothesised that continuous delivery of neurotrophic factor might be used to protect nerve fibres and prevent damage to nerve over several months of diabetes. We therefore tested the effectiveness of HSV-mediated expression of NT-3 driven by LAP2 in mice with 6 months of STZ-induced diabetes.

\section{Materials and methods}

Animals Experiments were conducted on 8-week-old male Swiss Webster mice weighing 25-30 g (Charles River, Wilmington, MA, USA) and in compliance with approved institutional animal care and use protocols. A total of 40 mice were rendered diabetic by injection of STZ $(100 \mathrm{mg} / \mathrm{kg}$ twice in 48 h) (Sigma-Aldrich, St Louis, MO, USA). Blood glucose levels were measured 2 weeks after STZ administration. Mice that had blood glucose levels $>16.6 \mathrm{mmol} / 1$ at 2 weeks were then divided into treatment groups. Blood glucose remained elevated throughout 6 months $(22.09 \pm 0.6 \mathrm{mmol} / 1$ in the diabetic mice compared with $5.96 \pm 0.8 \mathrm{mmol} / 1$ in control animals [means $\pm \mathrm{SEM}])$. These animals were not overtly ill, did not require insulin supplementation and by 6 months had an average weight that was reduced $<10 \%$ compared with controls (control $27.4 \pm 0.7 \mathrm{~g}$; diabetic $25.9 \pm 0.4 \mathrm{~g}$ ).

Subsets of ten mice were each inoculated s.c. into the plantar surface of both hind feet with $10 \mu \mathrm{l}$ containing $1 \times 10^{7}$ plaque forming units of HSV-based therapeutic vector expressing NT-3 (QLNT3) under the control of LAP2 [25], or a control vector expressing green fluorescent protein under the control of the HCMV IEp gene promoter and the lacZ gene under the control of HSV ICP0 (vector Q0ZHG). Ten animals that did not receive STZ served as normal controls, and another ten animals that received STZ but not 
the vectors were considered as the diabetic-only group. All analyses were carried out by an observer blinded to the treatment group.

Electrophysiology Electrophysiological recordings were performed on the right hind foot using a Nicolet Viking II (Nicolet Biomedical, Madison, WI, USA). Mice were anaesthetised with ketamine/xylazine $(80 / 10 \mathrm{mg} / \mathrm{kg}$ i.p.), the hind limbs were secured at an angle of $30^{\circ}$ relative to the body, and motor nerve conduction velocity and amplitude in the sciatic nerve was determined by inserting the recording electrode into the gastrocnemius muscle and the stimulating electrode pair at the sciatic notch or the knee; the reference electrode was inserted s.c. into the fifth digit of the hind limb. A ground electrode was inserted into the tail, and s.c. temperature was maintained at $36-37^{\circ} \mathrm{C}$. Both latency and baseline-to-peak amplitudes were determined.

For sensory nerve recordings, electrodes were inserted into the sciatic notch as the recording electrode, the stimulating electrode was placed at the ankle, and the reference electrode was positioned at the first digit. The statistical significance of the difference between groups was determined by ANOVA using the Bonferroni adjustment for multiple post-hoc analyses (Systat 9.0 software; SPSS, Chicago, IL, USA).

Sudomotor test Sudomotor function, reflecting innervation of sweat glands in the paw, is a measure to test autonomic function and was evaluated by quantifying the number of sweat droplets induced by injection of pilocarpine (SigmaAldrich) using the silicone imprint technique. Animals were injected with pilocarpine nitrate $(0.3 \mathrm{mg} / \mathrm{kg})$ to stimulate sweating. At $10 \mathrm{~min}$ after the injection, silicone (Silaplast and Silasoft; Detax, Ettlingen, Germany) was spread over the plantar surface of the hind paw and left in place for $10 \mathrm{~min}$. The mould was carefully removed, and the amount of sweating was determined by counting the number of sweat droplet impressions made in the silicone mould under a dissecting microscope.

Hotplate test Thermal pain sensation was assessed by a hotplate test. Animals were placed on a metal plate (Ugo Basile, Comeria, Italy) heated to $47^{\circ} \mathrm{C}$, and the temperature increased at $2^{\circ} \mathrm{C} / \mathrm{min}$. Withdrawal latency was measured in seconds to the time the animals lifted their paw from the plate or licked the paw [26].

Immunocytochemistry Mice were perfused transcardially with $9 \mathrm{~g} / \mathrm{l} \mathrm{NaCl}$ followed by Zamboni's fixative [27]. The spinal cord, DRG, plantar footpads and toes were removed, post-fixed with Zamboni's fixative for $2 \mathrm{~h}$, and then cryoprotected with $300 \mathrm{~g} / 1$ sucrose in PBS overnight. All tissues were cryostat sectioned at $10 \mu \mathrm{m}$, collected on gelatincoated slides, fixed with $20 \mathrm{~g} / \mathrm{l}$ paraformaldehyde for $15 \mathrm{~min}$, washed with PBS, and incubated with blocking solution (PBS with $10 \mathrm{ml} / 1$ normal goat serum and $3 \mathrm{ml} / 1$ Triton X-100) for $1 \mathrm{~h}$, then washed once. The DRG and spinal cord were incubated with the primary antibody, rabbit anti-calcitonin gene-related peptide (CGRP) at 1:1,000 dilution (Peninsula Laboratories, San Carlos, CA, USA), for $2 \mathrm{~h}$ at room temperature. The footpads were incubated with anti-protein gene product (PGP) 9.5 at 1:1,000 dilution (Chemicon International, Temecula, CA, USA) overnight at $4^{\circ} \mathrm{C}$ and washed three times. After incubation in the secondary fluorescent antibody, Alexa Fluor 594 goat anti-rabbit IgG at 1:1,000 dilution (Molecular Probes, Eugene, OR, USA), for $1 \mathrm{~h}$ at room temperature, the specimens were washed three times and mounted in water-based Fluoromount G (Electron Microscopy Sciences, Fort Washington, PA, USA).

Morphometric analysis Immunostained sections were viewed with an Olympus BX61 Fluoview laser-scanning microscope and the digitised images analysed using a PCbased image analysis program (MCID; Imaging Research, Brock, ON, Canada) to determine the area occupied by the immunostained sensory nerve terminals in the dorsal horn or the proportional area occupied by nerve fibres in the s.c. tissue of the footpad.

Cell culture DRG from 17 day Sprague-Dawley rat embryos (Charles River, Wilmington, MA, USA) were dissociated with $2.5 \mathrm{~g} / 1$ trypsin with $1 \mathrm{mmol} / 1$ EDTA for $30 \mathrm{~min}$ at $37^{\circ} \mathrm{C}$ with constant shaking and then plated on poly-D-lysine-coated coverslips at $10^{5}$ cells per well in a 24 well plate in $500 \mu \mathrm{l}$ defined Neurobasal medium containing B27, Glutamax I, Albumax II and penicillin/streptomycin (Gibco-BRL, Carlsbad, CA, USA), supplemented with $100 \mathrm{ng} / \mathrm{ml}$ 7.0S NGF per ml (Sigma). At 17 days in vitro, NGF was removed from the culture medium and $24 \mathrm{~h}$ later the cells were infected with QLNT3 or Q0ZHG. Cells were collected $24 \mathrm{~h}$ after the infection (three wells were pooled to one sample) and homogenised with lysis buffer $(20 \mathrm{mmol} / 1 \mathrm{Tris}, \mathrm{pH} 7.5$, $150 \mathrm{mmol} / 1 \mathrm{NaCl}, 1 \mathrm{mmol} / \mathrm{l}$ EDTA, $10 \mathrm{ml} / 1$ Triton X-100, $100 \mathrm{ml} / 1$ glycerol and 1:100 dilution of protease inhibitor cocktail and phosphatase inhibitor cocktail (Sigma). The lysed cells were then centrifuged at $10,000 \times \mathrm{g}$ for $10 \mathrm{~min}$ at $4^{\circ} \mathrm{C}$. An aliquot of supernatant was taken for protein estimation using a protein assay kit (Bio-Rad Laboratories, Hercules, CA, USA).

Western blot Total cell extract (20 $\mu \mathrm{g}$ protein per lane) was separated by PAGE, transferred to an Immobilon-P membrane (0.45 $\mu \mathrm{m}$; Millipore, Bedford, MA, USA), blocked with $50 \mathrm{~g} / 1$ non-fat milk, then incubated with the primary antibody (anti-phosphorylated high-affinity NGF receptor [TrkA]; Cell Signaling, Beverly, MA, USA), followed by horseradish peroxidase-conjugated anti-rabbit IgG (1:10,000; 
Sigma) and visualised with enhanced chemiluminescence (Amersham Biosciences, Piscataway, NJ, USA). The lower half of the membrane was probed with anti- $\beta$-actin $(1: 2,000$; Sigma) as a loading control. The intensity of each band was determined by quantitative chemiluminescence using a PC-based image analysis system (ChemiDoc XRS System; Bio-Rad).

RT-PCR of sympathetic ganglia In order to assess expression of the transgene in autonomic ganglia, L4-L6 DRG and paravertebral sympathetic ganglia were removed 7 days after vector inoculation and total RNA isolated using a commercial kit (MicroRNA; Stratagene, La Jolla, CA, USA). cDNA was transcribed from the mRNA at $37^{\circ} \mathrm{C}$ using a Sensiscript Reverse Transcriptase kit (Qiagen, Valencia, CA, USA), and RT-PCR completed using the following primers: forward, $5^{\prime}$ TTCTTTCAGATCTTACAGGTGAAC-3'; reverse 5'AATAATTTATATGTGGGGACAGAT- $3^{\prime}$ for 35 cycles $\left(94^{\circ} \mathrm{C}\right.$ for $1 \mathrm{~min}, 60^{\circ} \mathrm{C}$ for $1 \mathrm{~min}, 72^{\circ} \mathrm{C}$ for $1 \mathrm{~min}$ ), and the products separated on a $1.2 \%$ agarose gel.

Statistical analysis The statistical significance of the difference between groups was determined by ANOVA (Systat 9) using Bonferroni's correction for the multiple post hoc analyses. Results are expressed as means \pm SEM.

\section{Results}

Vector QLNT3 contains the human NT-3-coding sequence under control of the HSV LAP2 element in the $\mathrm{U}_{\mathrm{L}} 41$ locus of an HSV recombinant defective in expression of the HSV immediate early gene products ICP4, ICP22, ICP27 and ICP47 [25]. We have previously demonstrated that this vector produces NT-3 in vitro and in vivo, and that following transduction of DRG by s.c. inoculation the production of NT-3 in DRG measured by ELISA remains relatively unchanged over 6 months [25].

Vector-mediated NT-3 production preserves sensory nerve function in diabetic animals Diabetic animals demonstrated a marked decrease in amplitude of the evoked sensory nerve action potential compared with controls 1 month after diabetes (control $28.8 \pm 1.3 \mu \mathrm{V}$, diabetic $10.3 \pm 0.8 \mu \mathrm{V} ; p<$ 0.001 ANOVA). Animals transduced with QLNT3 2 weeks after diabetes demonstrated protection of sensory nerve amplitude 1 month after vector inoculation (QLNT3 26.3 \pm $1.1 \mu \mathrm{V}$, Q0ZHG $14.5 \pm 1.5 \mu \mathrm{V} ; p<0.001$, Fig. 1a). Animals tested 6 months after diabetes also showed reduced sensory nerve action potential, while QLNT3-inoculated animals had substantial preservation of sensory nerve amplitude (QLNT3 23.2 $\pm 1.8 \mu \mathrm{V}$, diabetic $12.0 \pm 0.9 \mu \mathrm{V} ; p<0.001$, Fig. 1b). Diabetic animals demonstrated a decrease in sensory nerve conduction velocity compared with controls (control $23.3 \pm 0.3 \mathrm{~m} / \mathrm{s}$, diabetic $20.3 \pm 0.3 \mathrm{~m} / \mathrm{s} ; p<0.01$, Fig. 1c). Animals transduced with QLNT3 2 weeks after diabetes demonstrated preservation of sensory nerve conduction velocity (QLNT3 $22.3 \pm 0.3 \mathrm{~m} / \mathrm{s} ; p<0.05$ compared with diabetic animals).

Preservation of motor nerve function in diabetic animals at 6 months by the NT-3-expressing vector At 6 months, diabetic animals had a significant reduction in motor nerve conduction velocity (control $51.6 \pm 1.4 \mathrm{~m} / \mathrm{s}$, diabetic $37.6 \pm 0.5 \mathrm{~m} / \mathrm{s} ; p<0.001)$. QLNT3-inoculated diabetic rats showed preservation of motor nerve conduction velocity compared with control vector Q0ZHG-inoculated animals (Fig. 2a) which showed a substantial decrease in conduction velocity, and were not significantly different from the diabetic-only group (QLNT3-inoculated diabetic $50 \pm 1.4 \mathrm{~m} / \mathrm{s}$, Q0ZHG-inoculated diabetic $39.8 \pm 1.2 \mathrm{~m} / \mathrm{s}$; $p<0.001)$.
Fig. 1 Preservation of sensory nerve amplitude and conduction velocity in QLNT3-treated diabetic animals at 6 months. Mice rendered diabetic by STZ and inoculated with QLNT3 2 weeks later showed preservation of sensory nerve amplitude measured at 1 month (a) and at 6 months (b) after inoculation of QLNT3, and also showed preservation of sensory nerve conduction velocity at 6 months (c). ${ }^{*} p<0.05, * * p<0.001, n=8-10$ animals per group, means \pm SEM
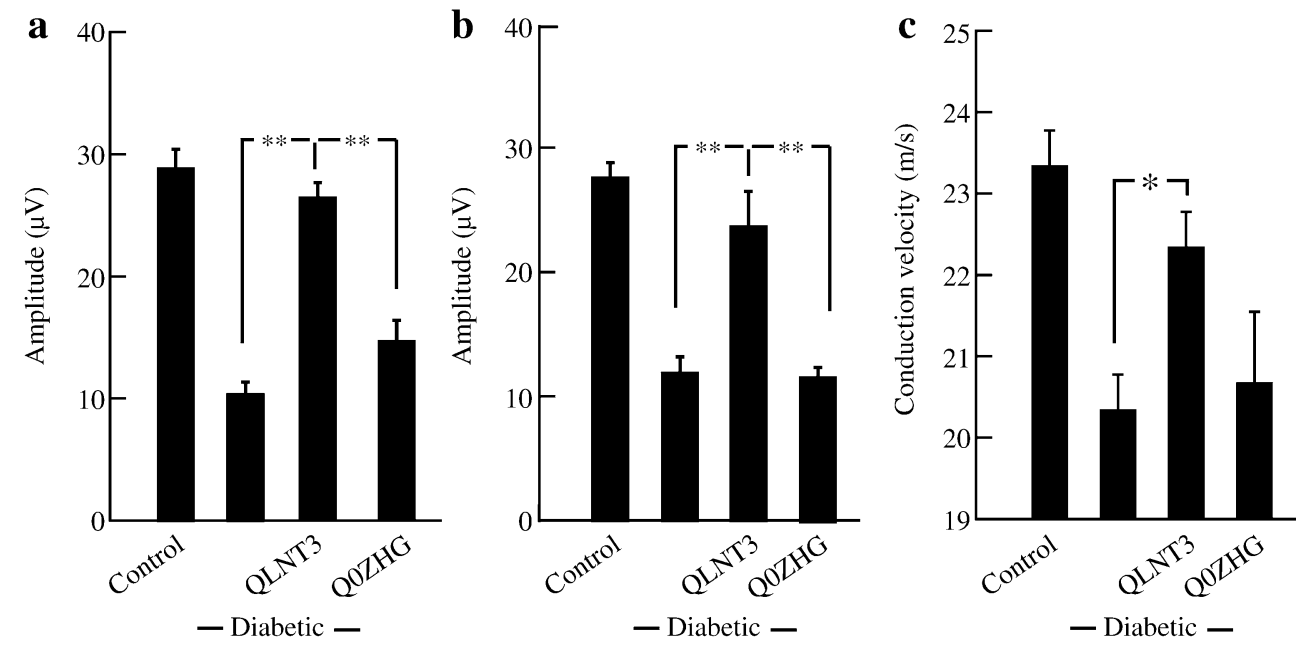

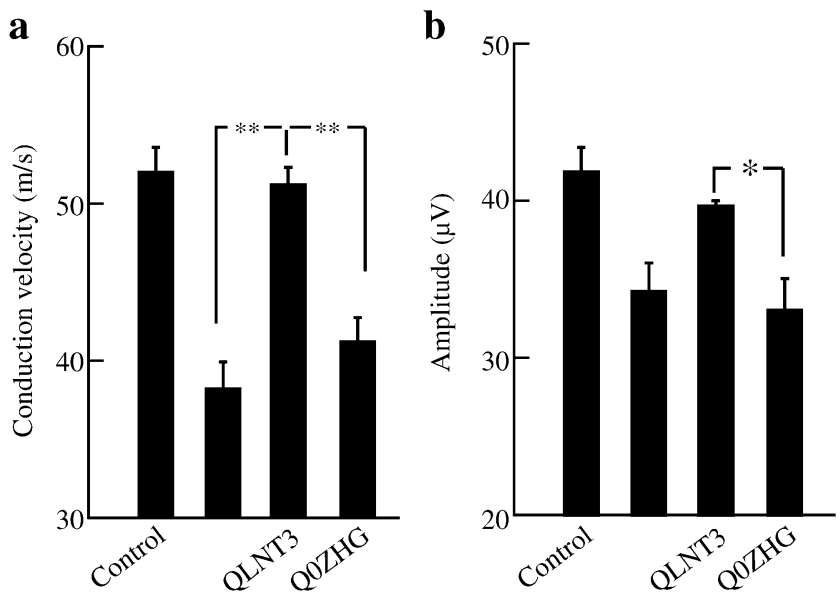

- Diabetic -

- Diabetic -

Fig. 2 Preservation of motor nerve functions after 6 months of STZdiabetic neuropathy. Mice rendered diabetic by STZ and inoculated with QLNT3 2 weeks later showed substantial preservation of motor nerve function measured by motor nerve conduction velocity (a) and amplitude (b). ${ }^{*} p<0.01,{ }^{* *} p<0.001, n=8-10$ animals per group, means \pm SEM

Motor nerve amplitude was also reduced in diabetic animals at 6 months. Like motor nerve conduction velocity, motor nerve amplitude was preserved in the QLNT3-treated diabetic animals (QLNT3-inoculated diabetic $38.8 \pm 0.5 \mathrm{mV}$, Q0ZHG-inoculated diabetic $32.4 \pm 1.5 \mathrm{mV} ; p<0.01$, Fig. 2b).

\section{Protection of thermal pain sensation by HSV-mediated} expression of NT-3 Diabetic animals tested 6 months after injection of STZ showed reduced thermal sensation manifested by an increased latency to withdraw (control $20.33 \pm 0.8 \mathrm{~s}$; diabetic $31 \pm 1.0 \mathrm{~s} ; p<0.0001$, ANOVA). Animals inoculated with QLNT3 2 weeks after STZ injection showed preservation of pain sensation, while control vector-inoculated animals were indistinguishable from the diabetic untreated group (latency $24 \pm 0.5 \mathrm{~s} ; p<$ 0.001 compared with Q0ZHG, Fig. 3).

Fig. 3 Vector effects on small fibre (pain) sensory function. Thermal withdrawal (latency, in s) was substantially preserved in diabetic animals inoculated with QLNT3 compared with diabetic controls tested after 6 months of diabetes. ${ }^{* *} p<0.001, n=8-10$ animals per group

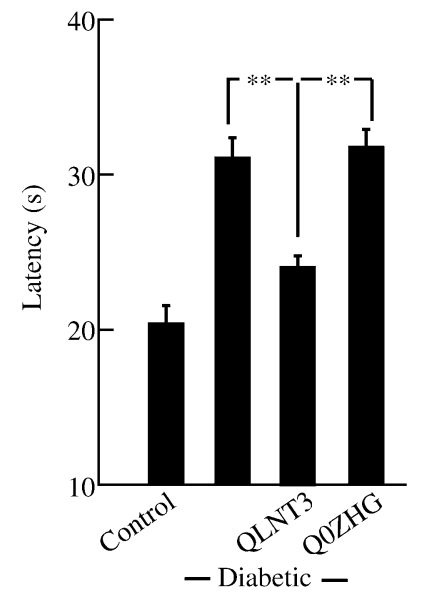

Sudomotor function preserved by HSV-mediated expression of NT-3 Autonomic innervation of sweat glands in the paw was evaluated by measuring the number of sweat droplets formed over $10 \mathrm{~min}$ after injection of pilocarpine. Reduction
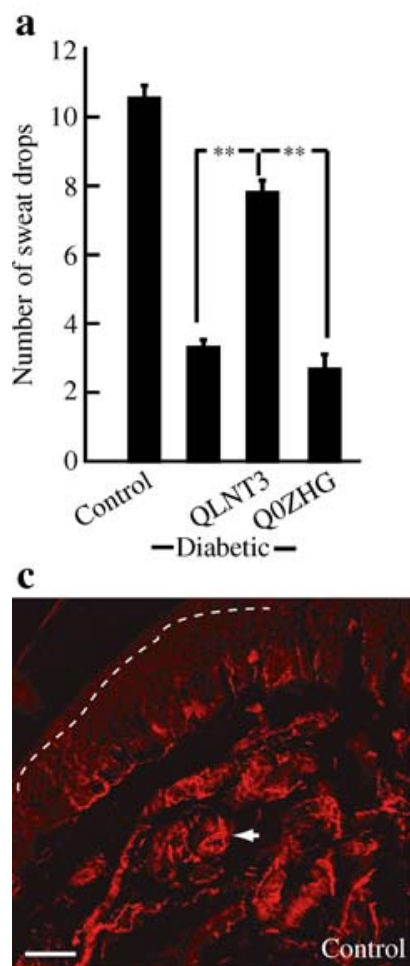

d
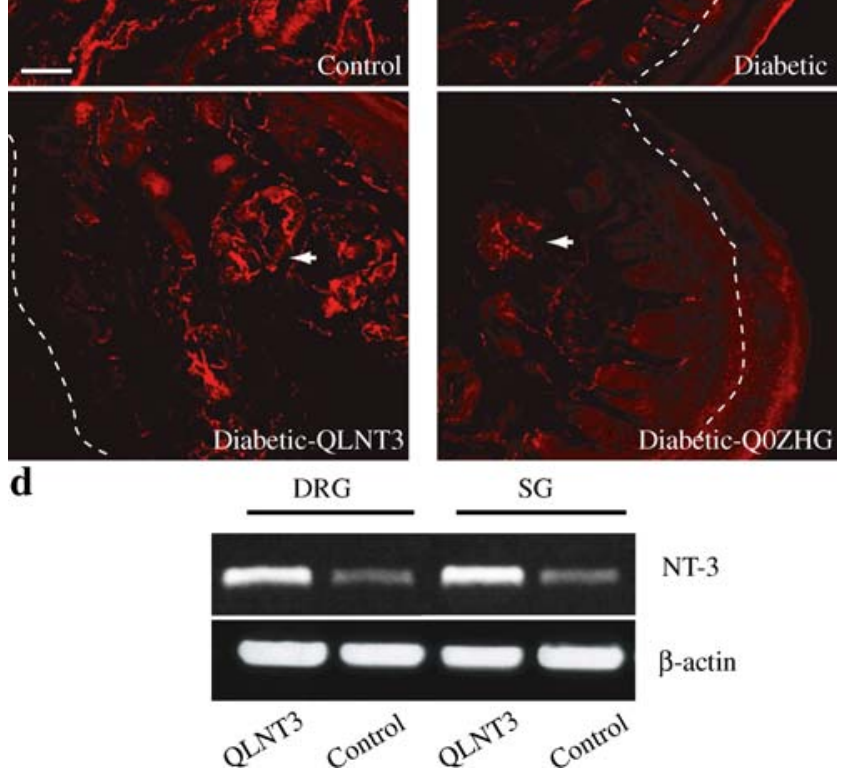

Fig. 4 a Pilocarpine-induced sweating (number of drops per sweat gland) was substantially preserved in diabetic animals inoculated with QLNT3 compared with diabetic controls tested after 6 months of diabetes. Innervation of the sweat glands in the foot was similarly preserved: b proportional area; c photomicrograph (arrows indicate sweat glands in animals inoculated with QLNT3 compared with diabetic controls tested after 6 months of diabetes. The outer edge of the epidermis is indicated by a dotted line; bar $=50 \mu \mathrm{m} .{ }^{*} p<0.005$, $* * * p<0.0001, n=4-5$ animals per group, means \pm SEM. d RT-PCR of NT-3 in DRG and sympathetic ganglia $(S G)$ from QLNT3-inoculated and control mice killed 1 week after inoculation. A representative blot is shown with $\beta$-actin as internal control 
in the number of sweat droplets in diabetic mice reflecting autonomic neuropathy (control $10.5 \pm 0.2$ droplets; diabetic $3.3 \pm 0.1$ droplets; $p<0.0001)$ was prevented in animals inoculated with therapeutic vector (QLNT3 $7.5 \pm 0.2$ droplets; Q0ZHG $2.6 \pm 0.3$ droplets; $p<0.005$, Fig. 4a). The reduction in pilocarpine-induced sweat production correlated with a marked decrease in PGP 9.5-immunoreactive fibres surrounding the sweat glands in diabetic animals (control $0.052 \pm 0.004$; diabetic $0.014 \pm 0.004 ; p<0.0001$; Fig. $4 b, c)$. Diabetic animals inoculated with QLNT3 showed significant preservation of nerve terminals in the sweat gland of the footpad while control vector-treated animals showed no preservation of innervation (QLNT3 $0.035 \pm 0.005$; Q0ZHGinoculated diabetic $0.009 \pm 0.003 ; p<0.0001$; Fig. $4 \mathrm{~b}, \mathrm{c})$. The reduction in pilocarpine-induced sweat production correlated with a marked decrease in PGP 9.5-immunoreactive fibres surrounding the sweat glands in diabetic animals, which appeared to be preserved in diabetic QLNT3inoculated but not in diabetic Q0ZHG-inoculated mice (Fig. 4b,c). NT-3 RNA could be detected by RT-PCR in sympathetic ganglia from rats inoculated with QLNT3 (Fig. 4d) in amounts roughly similar to those found in transduced DRG. No band was detected in the without-RT control (data not shown).

Preservation of distal innervation of skin by LAP2-driven NT-3 production Distal innervation of the toe and plantar surface footpad after 6 months of diabetes was visualised by PGP 9.5 staining and quantified by determining the fraction of the s.c. surface taken up by PGP 9.5-immunoreactive fibres. In control animals, nerve fibres were found principally in dermis with a few fine fibres extending into the epidermis, as has been described previously [28]. In diabetic animals, whether untreated or inoculated with control vector Q0ZHG, very few nerve fibres were detected

Fig. 5 PGP 9.5-immunoreactive fibres in the skin from toes and plantar footpads. a Innervation in the footpad was quantified by the area occupied by PGP 9.5-immunoreactive fibres. $* * * p<0.0001, n=5$ animals per group, means \pm SEM. b Representative images of sections from toe skin in each group are shown. The location of the epidermis is indicated by the letter 'e'; nerve fibres by arrows; bar $=50 \mu \mathrm{m}$ in the dermis and the proportional area occupied by nerve fibres was markedly reduced (control $0.056 \pm 0.001$; diabetic $0.006 \pm 0.003 ; p<0.0001)$. In contrast, diabetic mice inoculated with QLNT3 2 weeks after injection of STZ showed extensive preservation of innervation after 6 months of diabetes (QLNT3-inoculated diabetic proportional area $0.057 \pm 0.005$; Q0ZHG-inoculated diabetic $0.008 \pm 0.003$; $p<$ 0.0001 , Fig. 5).

Preservation of neuropeptide levels in afferent terminals in the spinal cord of diabetic mice We assessed neuropeptide production in small-diameter afferents by determining the area of CGRP immunoreactivity in the superficial laminae of the dorsal horn of the spinal cord. The area of CGRP immunoreactivity in diabetic mice was reduced compared with control animals, consistent with the loss of unmyelinated fibre afferents into the dorsal horn (control $5.5 \pm 0.2 \mu^{2}$, diabetic $2.5 \pm 0.2 \mu \mathrm{m}^{2} ; p<$ 0.0001). Diabetic animals inoculated with QLNT3 showed preservation of CGRP production (QLNT3-inoculated diabetic $4.5 \pm 0.2 \mu^{2}$; Q0ZHG-inoculated diabetic $3.0 \pm$ $0.4 ; p<0.005$, Fig. 6).

NT-3 activates TrkA in primary DRG neurons in vitro The effects of the vector on small sensory and autonomic fibres extend beyond what are conventionally deemed pure NT-3 effects. In order to determine whether vector-produced NT3 might activate TrkA, we infected primary DRG neurons in culture with QLNT3 for $24 \mathrm{~h}$ in the absence of NGF and assessed the phosphorylation of TrkA using a specific antiphosphorylated TrkA antibody. Cells transduced with QLNT3, but not cells transduced with control vector Q0ZHG, showed phosphorylation of TrkA (Fig. 7), indicating that NT-3 alone is capable of activating TrkA.

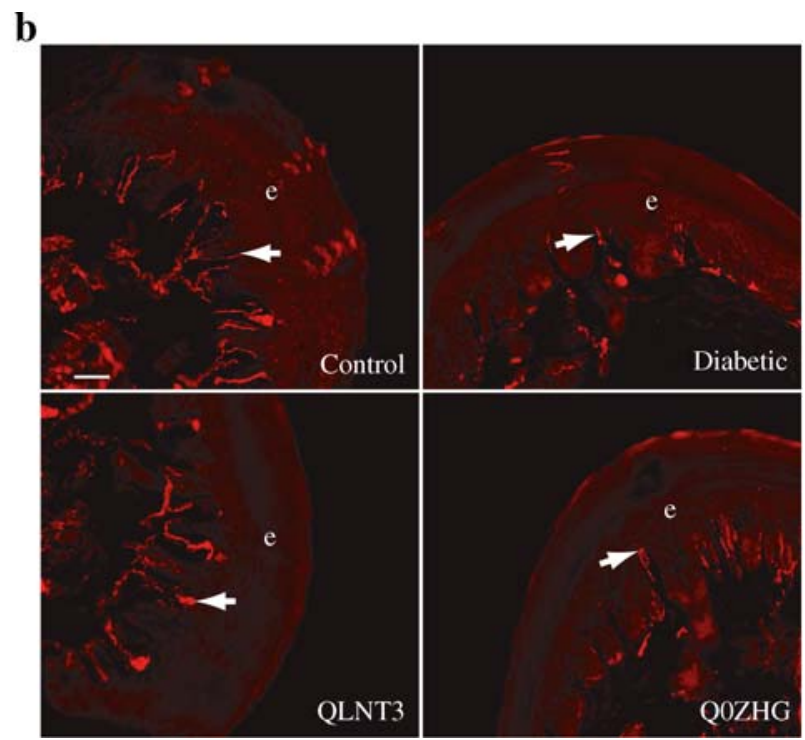


Fig. 6 Inoculation with QLNT3 preserved CGRP-immunoreactive fibres in lamina I and II in the dorsal horn of spinal cord in diabetic animals. a CGRP expression was quantified by the area of the dorsal horn occupied by CGRP-immunoreactive $(C G R P-I R)$ fibres. $* * p<0.005$, $* * * p<0.0001, n=5$ animals per group, means \pm SEM. b Representative images of spinal cord sections are shown. $B a r=50 \mu \mathrm{m}$

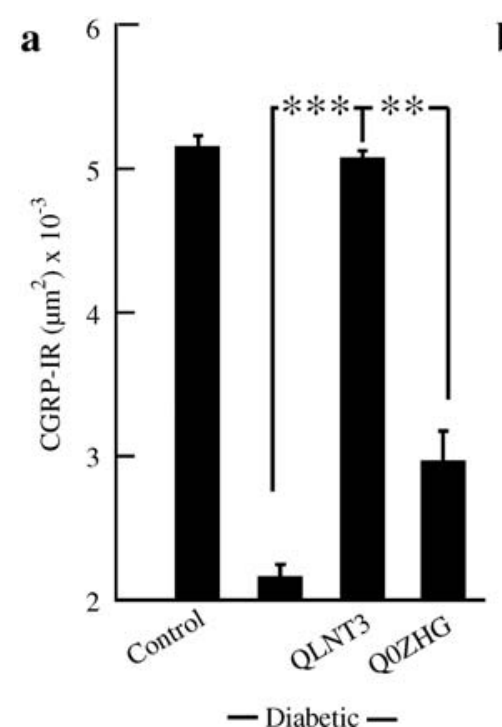

b

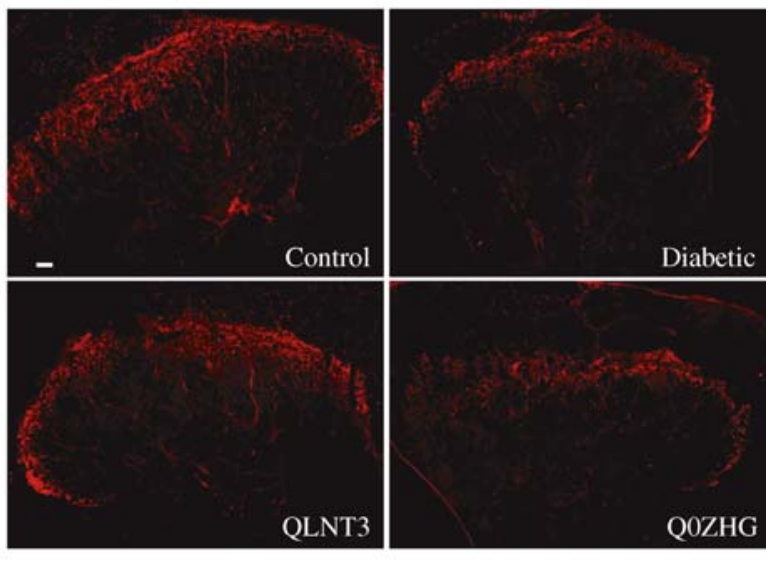

\section{Discussion}

The results of this study demonstrate that continuous delivery by LAP2-driven expression of NT-3 from a nonreplicating HSV vector effectively protects against the development of neuropathy in mice with STZ-induced diabetes over the course of 6 months. In previous work we

a

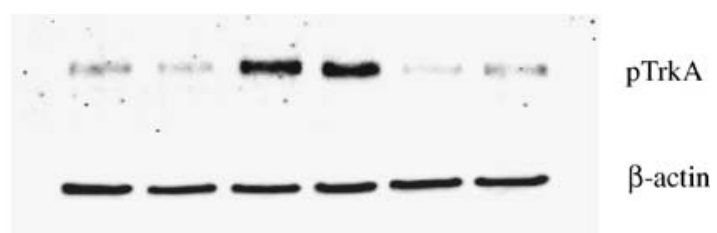

b

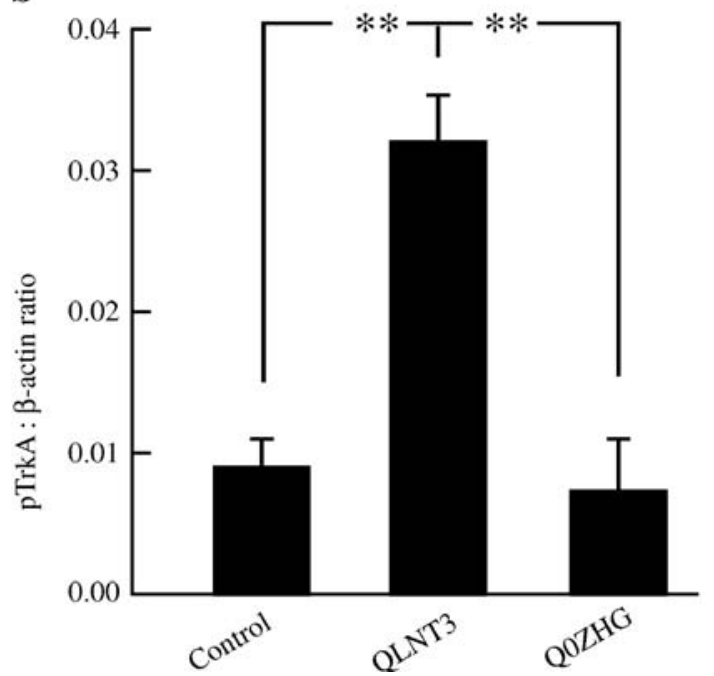

Fig. 7 NT-3 produced by QLNT3 phosphorylates TrkA in vitro. a Western blot of phosphorylated TrkA ( $p$ TrkA) $24 \mathrm{~h}$ after transfection with QLNT3 or control vector Q0ZHG. b pTrkA: $\beta$-actin ratio. ${ }^{* *} p<$ 0.001 comparing QLNT3 with Q0ZHG or with control, $n=3$ per group, means \pm SEM determined that the LAP2 element is capable of driving prolonged expression in DRG neurons in vivo, and that LAP2-driven expression of NT-3 is sufficient to protect against neuropathy caused by an acute dose of pyridoxine administered over a course of 8 days beginning 5 1/2 months after vector inoculation [25]. In diabetes the pathogenic process is sustained over the course of several months; the ability of the LAP2 NT-3 vector to protect nerve structure and function over that prolonged time course indicates a continuous therapeutic effect with a duration $>5$ months.

The HSV LAP2 element (nucleotides 118,866-119,461 of the HSV genome) is a moveable sequence that naturally drives the long-term expression of latency-associated transcripts (LATs) in latently infected DRG neurons in vivo [29]. In humans, LAT expression persists for decades [30]. We have previously demonstrated that this promoter is effective in driving expression of a transgene from an HSV vector in rodent brain [24] and in rodent DRG [25] over the course of 6 months. While the amount of transgene expression driven by LAP2 is less than that achieved by the stronger HCMV IEp, the results of the current study indicate that the amount produced in DRG neurons is sufficient to protect against the progression of diabetic neuropathy. The results we obtained are complementary to results that have been reported in a genetic model of type 2 diabetes in the mouse, transduced with a non-replicating HSV vector containing a similar, but not identical, latencyrelated promoter construct [31].

In the current study we observed that NT-3 production preserved the production of CGRP in the DRG of diabetic animals and preserved autonomic function measured by pilocarpine-induced sweating. CGRP is produced in unmyelinated fibres of small-diameter DRG neurons, cells such as sympathetic neurons that produce high levels of the high-affinity TrkA receptor, but are not conventionally considered to respond to the TrkC ligand NT-3. There are 
several possible explanations for this finding. It is known that DRG neurons can switch neurotrophin dependency during development [32], and that in some situations NT-3 can act through the non-selective low-affinity (p75) neurotrophin receptor to achieve protective effect [33]. We found in our experiments that NT-3 produced from the vector results in phosphorylation of the TrkA high-affinity NGF receptor in primary DRG neurons in culture. This effect may relate to the concentration of NT-3 available at the receptor but may also depend on the level of co-expression of the p75 receptor. In the absence of p75 the NT-3 responsiveness of TrkA is increased [34], and a sequential response of developing sympathetic neurons to NT-3 followed by NGF appears to be related to the level of p75 production in those cells [35] and can be demonstrated in the phenotype of NT-3-overexpressing mice [36]. A second possibility is that the effect is mediated through the relatively low level of TrkC that is produced in sympathetic ganglia [30, 31]. Finally, we cannot exclude the possibility that NT-3 production might induce the production of NGF in this system, although that would be less likely.

The protection of autonomic fibres would appear probably to result from the delivery of the vector to the cell bodies of autonomic ganglia through retrograde axonal transport, as we detected the expression of mRNA transcripts for NT-3 in the autonomic ganglia by RT-PCR. Whether NT-3 released from transduced sensory neuron terminals centrally or peripherally plays a role in this effect has not been established.

In other studies it has been shown that NT-3 can reverse established sensory and motor conduction velocity deficits in diabetic STZ rats when administration was started after 8 weeks of diabetes [7], and treatment started after 4 weeks of diabetes could reverse abnormalities in neurofilaments related to diabetes [8]. However, prolonged diabetes results in other changes in peripheral nerve [37], including ultimately the loss of DRG neurons, that are likely to be irreversible [38].

Protective effects of gene transfer in diabetic neuropathy models of shorter duration in rats and rabbits have been achieved by the i.m. injection of plasmids or other viral vectors $[12,16]$. In those approaches, protection is achieved by the systemic release of the peptide factor from transduced muscle. Although no adverse effects were observed in those models, there are two issues regarding the scale-up to human treatment. The amount of vector required for i.m. inoculation would need to be scaled up according to volume, which may result in a prohibitive requirement for vector dosing into muscle. Second, longterm production of these peptides reaching the systemic circulation may result in adverse effects on other receptorbearing cells. In that regard, direct expression within DRG neurons would appear to be a preferable choice.
In this model of diabetic neuropathy, we demonstrated that vector inoculation 2 weeks after the onset of diabetes is capable of preventing the development of diabetic neuropathy over the course of 6 months. Although in this model we have not reversed an established diabetic neuropathy, we believe that this approach nonetheless represents a treatment that would be appropriate for human disease. Once the neuropathy progresses to the point that skin innervation has been lost, HSV-mediated gene transfer from the skin is not likely to be effective. However, because diabetic neuropathy characteristically presents as a subacute to chronic progressive condition beginning distally, administration of a vector after the onset to prevent the progression of neuropathy would represent a substantial advance over available treatments that at best slow the progression of neuropathy. We conclude that HSV-mediated NT-3 production driven by LAP2 provides a sustained effect that is likely to be effective for the long-term treatment of diabetic neuropathy. The principal outstanding issue to be addressed is the development of a regulatable system to control gene expression so that if an adverse event (resulting from activation of TrkA, for example) were to occur, transgene expression could be shut off.

Acknowledgements We acknowledge the excellent technical assistance of V. S. Thakur in propagation of the vectors employed in this study, C. Fetteroff and A. Tubio in the animal experiments and S. Liu for primary DRG culture. This work was supported by research grants from the Juvenile Diabetes Research Foundation, the National Institutes of Health and the Department of Veterans Affairs.

Duality of interest D. Wolfe is currently an employee of Diamyd, and J. C. Glorioso holds an equity interest in and serves as a consultant for Diamyd. The other authors declare no duality of interest.

\section{References}

1. Sullivan KA, Feldman EL (2005) New developments in diabetic neuropathy. Curr Opin Neurol 18:586-590

2. Low PA, Nickander KK, Tritschler HJ (1997) The roles of oxidative stress and antioxidant treatment in experimental diabetic neuropathy. Diabetes 46(Suppl 2):S38-S42

3. Tomlinson DR, Fernyhough P, Diemel LT (1997) Role of neurotrophins in diabetic neuropathy and treatment with nerve growth factors. Diabetes 46(Suppl 2):S43-S49

4. Fernyhough P, Diemel LT, Tomlinson DR (1998) Target tissue production and axonal transport of neurotrophin-3 are reduced in streptozotocin-diabetic rats. Diabetologia 41:300-306

5. Cai F, Tomlinson DR, Fernyhough P (1999) Elevated expression of neurotrophin-3 mRNA in sensory nerve of streptozotocindiabetic rats. Neurosci Lett 263:81-84

6. Kennedy AJ, Wellmer A, Facer P et al (1998) Neurotrophin-3 is increased in skin in human diabetic neuropathy. J Neurol Neurosurg Psychiatry 65:393-395

7. Mizisin AP, Calcutt NA, Tomlinson DR, Gallagher A, Fernyhough P (1999) Neurotrophin-3 reverses nerve conduction velocity deficits in streptozotocin-diabetic rats. J Peripher Nerv Syst 4:211-221 
8. Sayers NM, Beswick LJ, Middlemas A et al (2003) Neurotrophin3 prevents the proximal accumulation of neurofilament proteins in sensory neurons of streptozocin-induced diabetic rats. Diabetes 52:2372-2380

9. Middlemas A, Delcroix JD, Sayers NM, Tomlinson DR, Fernyhough P (2003) Enhanced activation of axonally transported stress-activated protein kinases in peripheral nerve in diabetic neuropathy is prevented by neurotrophin-3. Brain 126:1671-1682

10. Apfel SC, Arezzo JC, Brownlee M, Federoff H, Kessler JA (1994) Nerve growth factor administration protects against experimental diabetic sensory neuropathy. Brain Res 634:7-12

11. Bianchi R, Buyukakilli B, Brines M et al (2004) Erythropoietin both protects from and reverses experimental diabetic neuropathy. Proc Natl Acad Sci USA 101:823-828

12. Schratzberger P, Walter DH, Rittig K et al (2001) Reversal of experimental diabetic neuropathy by VEGF gene transfer. J Clin Invest 107:1083-1092

13. Apfel SC (2002) Nerve growth factor for the treatment of diabetic neuropathy: what went wrong, what went right, and what does the future hold? Int Rev Neurobiol 50:393-413

14. Thoenen H, Sendtner M (2002) Neurotrophins: from enthusiastic expectations through sobering experiences to rational therapeutic approaches. Nat Neurosci 5(Suppl):1046-1050

15. Pradat PF, Finiels F, Kennel P et al (2001) Partial prevention of cisplatin-induced neuropathy by electroporation-mediated nonviral gene transfer. Hum Gene Ther 12:367-375

16. Pradat PF, Kennel P, Naimi-Sadaoui S et al (2001) Continuous delivery of neurotrophin 3 by gene therapy has a neuroprotective effect in experimental models of diabetic and acrylamide neuropathies. Hum Gene Ther 12:2237-2249

17. Pradat PF, Kennel P, Naimi-Sadaoui S et al (2002) Viral and nonviral gene therapy partially prevents experimental cisplatininduced neuropathy. Gene Ther 9:1333-1337

18. Chattopadhyay M, Wolfe D, Huang S et al (2002) In vivo gene therapy for pyridoxine-induced neuropathy by herpes simplex virus-mediated gene transfer of neurotrophin-3. Ann Neurol 51:19-27

19. Chattopadhyay M, Goss J, Lacomis D et al (2003) Protective effect of HSV-mediated gene transfer of nerve growth factor in pyridoxine neuropathy demonstrates functional activity of trkA receptors in large sensory neurons of adult animals. Eur $\mathrm{J}$ Neurosci 17:732-740

20. Chattopadhyay M, Goss J, Wolfe D et al (2004) Protective effect of herpes simplex virus-mediated neurotrophin gene transfer in cisplatin neuropathy. Brain 127:929-939

21. Goss JR, Goins WF, Lacomis D et al (2002) Herpes simplexmediated gene transfer of nerve growth factor protects against peripheral neruropathy in streptozotocin-induced diabetes in the mouse. Diabetes 51:2227-2232

22. Chattopadhyay M, Krisky D, Wolfe D et al (2005) HSV-mediated gene transfer of vascular endothelial growth factor to dorsal root ganglia prevents diabetic neuropathy. Gene Ther 12:1377-1384
23. Glorioso JC, Fink DJ (2004) Herpes vector-mediated gene transfer in treatment of diseases of the nervous system. Annu Rev Microbiol 58:253-271

24. Puskovic V, Wolfe D, Goss J et al (2004) Prolonged biologically active transgene expression driven by HSV LAP2 in brain in vivo. Mol Ther 10:67-75

25. Chattopadhyay M, Wolfe D, Mata M et al (2005) Long-term neuroprotection achieved with latency-associated promoter-driven herpes simplex virus gene transfer to the peripheral nervous system. Mol Ther 12:307-313

26. Aloe L, Moroni R, Angelucci F, Fiore M (1997) Role of TNFalpha but not NGF in murine hyperalgesia induced by parasitic infection. Psychopharmacology (Berl) 134:287-292

27. Verdu E, Vilches JJ, Rodriguez FJ et al (1999) Physiological and immunohistochemical characterization of cisplatin-induced neuropathy in mice. Muscle Nerve 22:329-340

28. Ma W, Bisby MA (2000) Calcitonin gene-related peptide, substance $\mathrm{P}$ and protein gene product 9.5 immunoreactive axonal fibers in the rat footpad skin following partial sciatic nerve injuries. J Neurocytol 29:249-262

29. Goins WF, Sternberg LR, Croen KD et al (1994) A novel latencyactive promoter is contained within the herpes simplex virus type 1 UL flanking repeats. J Virol 68:2239-2252

30. Croen KD, Ostrove JM, Dragovic LJ, Smialek JE, Straus SE (1987) Latent herpes simplex virus in human trigeminal ganglia: Detection of an immediate early gene 'anti-sense' transcript by in situ hybridization. N Engl J Med 317:1427-1432

31. Walwyn WM, Matsuka Y, Arai D et al (2006) HSV-1-mediated NGF delivery delays nociceptive deficits in a genetic model of diabetic neuropathy. Exp Neurol 198:260-270

32. White FA, Silos-Santiago I, Molliver DC et al (1996) Synchronous onset of NGF and TrkA survival dependence in developing dorsal root ganglia. J Neurosci 16:4662-4672

33. Chen WP, Chang YC, Hsieh ST (1999) Trophic interactions between sensory nerves and their targets. J Biomed Sci 6:79-85

34. Brennan C, Rivas-Plata K, Landis SC (1999) The p75 neurotrophin receptor influences NT-3 responsiveness of sympathetic neurons in vivo. Nat Neurosci 2:699-705

35. Kuruvilla R, Zweifel LS, Glebova NO et al (2004) A neurotrophin signaling cascade coordinates sympathetic neuron development through differential control of TrkA trafficking and retrograde signaling. Cell 118:243-255

36. Albers KM, Perrone TN, Goodness TP et al (1996) Cutaneous overexpression of NT-3 increases sensory and sympathetic neuron number and enhances touch dome and hair follicle innervation. $\mathrm{J}$ Cell Biol 134:487-497

37. Scott JN, Clark AW, Zochodne DW (1999) Neurofilament and tubulin gene expression in progressive experimental diabetes: failure of synthesis and export by sensory neurons. Brain 122:2109-2118

38. Kennedy JM, Zochodne DW (2005) Experimental diabetic neuropathy with spontaneous recovery: is there irreparable damage? Diabetes 54:830-837 